\title{
INTRODUCCIÓN DEL PINO PIÑONERO (Pinus pinea L.) EN CHILE
}

\author{
Loewe, Verónica ${ }^{6}$, Delard, Claudia ${ }^{4}$, González, Marlene ${ }^{4}$ \\ Mutke, Sven ${ }^{7}$ y Fuentes, Verónica ${ }^{4}$.
}

\section{RESUMEN}

Se analiza el comportamiento de 6 procedencias europeas de pino piñonero (Lombardía, Toscana, Eslovenia, Meseta Castellana, Andalucía Occidental y Sierra Morena) en un sector de la zona central de Chile, y se relaciona su desarrollo con clases de sitio españolas.

También se analiza el impacto de factores climáticos y de diferentes prácticas de manejo.

Los resultados muestran que las procedencias españolas presentan una mejor adaptación, y que la especie presenta una gran potencialidad en la zona estudiada.

Palabras clave: Ensayo de procedencia, especie exótica, pino piñonero, Pinus pinea.

\section{SUMMARY}

Six European Stone Pine provenances (Lombardy, Tuscany, Slovenia, Meseta Castellana, Western Andalusia and Sierra Morena) behavior in central Chile, relating its development to Spanish site classes, was analyzed.

It was also analyzed the impact of climatic factors and different silviculture practices.

Results show that Spanish origins present a better adjustment, and that the species presents a great potential in the studied zone.

Key words: Introduced species, Mediterranean Stone Pine Pinus pinea, Provenance trial.

\footnotetext{
${ }^{6}$ Instituto Forestal (INFOR), Sede Metropolitana. Sucre 2397, Ñuñoa, Santiago, Chile. vloewe@infor.cl

${ }^{7}$ Instituto de Gestión Forestal Sostenible (SFM-RI), CIFOR-INIA. Ctra. a Coruña, km 7,5. 28040 Madrid.
} 


\section{INTRODUCCIÓN}

El pino piñonero, Pinus pinea L., es originario de la cuenca mediterránea; sus masas más extensas se sitúan en la Península lbérica, donde supera el medio millón de hectáreas, lo que equivale a tres cuartas partes de su distribución total. En su área de origen destaca por su papel colonizador y estabilizador de suelos con perfiles incipientes o erosionados, especialmente en arenales costeros y continentales, y por el aprovechamiento que se da a su semilla comestible, los piñones, ubicados dentro de las piñas. Se ha constatado la recolección de piñas de Pinus pinea desde el Paleolítico en el área mediterránea y en la actualidad constituye el principal producto de los pinares (Thirgood, 1981; Prada et al., 1997; Gil, 1999; Badal, 2001).

En Chile, esta especie fue introducida como ornamental por inmigrantes europeos (españoles e italianos), aunque no se la utilizó como árbol forestal hasta principios del siglo XX, cuando en 1912 Federico Albert, profesional alemán, bachiller en botánica y doctor en ciencias naturales contratado por el Gobierno de Chile, emprendió un interesante y exitoso programa de estabilización de dunas costeras, empleando numerosas latifoliadas y coníferas, entre ellas el pino piñonero, aparte del pino radiata y una serie de eucaliptos (Albert, 1909). Posteriormente Albert se quedó en Chile haciendo una contribución significativa al desarrollo forestal del país, donde es considerado un precursor de la conservación de recursos naturales y la ecología. Muchos de los ejemplares de pino piñonero que se establecieron entonces en las dunas aún están vivos a pesar de la elevada densidad empleada $(1 \times 1 \mathrm{~m} .1,5 \times 1,5 \mathrm{~m})$ y la competencia de ejemplares de otras especies colindantes, y también es posible encontrar numerosos ejemplares aislados en fundos cercanos, presentando dimensiones considerables con diámetros superiores a $1 \mathrm{~m}$.

Posteriormente, entre los años 1955 y 1962 se realizó un programa de cooperación técnica denominado "Plan de Desarrollo Agrícola e Higiene Rural de Maule, Nuble y Concepción", más conocido como "Plan Chillán", que contó con la colaboración del Programa de Cooperación Técnica de Estados Unidos de América, y cuyo objetivo fue lograr un desarrollo integral de las tres provincias, dándose prioridad a labores de fomento agrícola (Anónimo, 1957). Una de las especies promovidas por dicho programa fue el pino piñonero, el que se estableció a lo largo de la zona en grupos de 25 individuos sobre pastizales, con el objetivo de proporcionar sombra al ganado. Actualmente se encuentran muchos árboles aislados y pequeños grupos de tamaño variable, que por su edad serían los remanentes de dichas forestaciones. En esa zona los habitantes cosechan y comen piñones de la especie, los que provendrían de los árboles resultantes de dicha actividad; además se comercializan en los mercados principales del área (Chillán, Yungay). Más tarde, en 1967 el Departamento Forestal de la Dirección General de Agricultura y Pesca realizó una siembra aérea con la especie en la zona precordillerana de Nuble y Bío Bío, cuyos resultados de prendimiento no fueron evaluados formalmente.

En los años noventa se volvía a estudiar el potencial del pino piñonero y del algarrobo europeo Ceratonia siliqua L. como cultivos alternativos en la zona de clima mediterráneo de Chile. Frente a la sensibilidad a las heladas del algarrobo europeo, se constató la buena adaptación autoecológica del pino en la región. En 1998 se realizó un estudio sobre áreas potenciales para el cultivo de la especie en el país, identificándose 1,3 millones de hectáreas disponibles en terrenos desocupados, considerando una altitud límite de 1.000 msnm (Loewe y González, 2003). Con posterioridad se ha observado que dicha altitud es superada en varias regiones geográficas del país, con presencia de la especie hasta más de $2.000 \mathrm{msnm}$ al norte de Santiago; asimismo, las restricciones en suelos arcillosos parecen menores a las indicadas por la bibliografía sobre la especie.

Pinus pinea, denominado "piñonero europeo" para diferenciarlo del pehuén o "piñonero" nativo Araucaria araucana, presenta múltiples atractivos para Chile, dadas sus características de producción de madera y, sobre todo, de uno de los frutos secos más caros del mercado internacional (Loewe y González, 2007; Soto et al., 2008), convirtiéndose en un cultivo interesante para pequeños y medianos propietarios y campesinos que requieren obtener ingresos anuales; a la vez permitiría crear valor al rubro de la fruticultura mediante la generación de un nuevo producto 
factible de ser producido en terrenos pobres, erosionados y con una relativa baja disponibilidad hídrica respecto a los cultivos tradicionales empleados en la fruticultura; incluso se considera factible que Chile pudiera certificar su producción de piñones como producto orgánico, dadas las características de su cultivo y procesamiento y la ausencia de las plagas endémicas de sus países de origen, evitando las fumigaciones.

Dadas estas razones, el Instituto Forestal (INFOR), organismo ligado al Ministerio de Agricultura chileno, se encuentra ejecutando un proyecto titulado "El piñón comestible del pino piñonero (Pinus pinea L.), un negocio atractivo para Chile", cuyo objetivo es desarrollar y promover las bases para la producción del piñón del pino mediterráneo con fines de exportación. Esta iniciativa, en ejecución entre los años 2008 y 2012, diseña, implementa y evalúa modelos productivos para producir piñones de pino; evalúa la productividad y calidad del piñón producido en Chile; evalúa el potencial del mercado europeo para piñones de pino producidos en Chile determinando estrategias comerciales de acuerdo a los requerimientos del mercado internacional; evalúa la situación fitosanitaria de la especie en Chile; y analiza los impactos socioeconómicos y ambientales de la implementación del cultivo de este pino.

Uno de los estudios realizados sobre el desarrollo de la especie en Chile corresponde a la evaluación de un ensayo de procedencias establecido el año 1994 en la zona central de Chile, típicamente mediterránea, cuyos resultados se exponen en el presente trabajo.

\section{MATERIAL Y MÉTODO}

En 1992, INFOR inició en el marco del proyecto "Silvicultura de especies no tradicionales, una mayor diversidad productiva" la producción de plantas de pino piñonero en vivero, con el propósito de establecer un ensayo de procedencias y evaluar la adaptación y crecimiento de la especie, comparando diferentes procedencias europeas en condiciones de secano de la zona central de Chile.

En este ensayo se incluyó seis procedencias, tres del oeste y tres del centro de la cuenca mediterránea (Cuadro $\mathrm{N}^{\circ} 1$ ). Además de estos dos grupos de procedencias europeas, se había incluido originalmente material de una introducción local chilena de origen desconocido situada en Cauquenes, que no llegó a plantarse en campo por su baja tasa de germinación en vivero (Loewe et al., 1998). Para todos los casos, se utilizó como tratamiento pre-germinativo remojo en agua por 24 horas, obteniéndose las primeras plantas a partir de la segunda semana después de la siembra.

\section{Cuadro $\mathrm{N}^{\circ} 1$}

PROCEDENCIAS ENSAYADAS (3 CISALPINAS Y 3 IBÉRICAS)

\begin{tabular}{|c|c|c|c|c|c|}
\hline & Procedencia & Localización & $\begin{array}{c}\text { PP } \\
\text { Media } \\
\text { Anual } \\
(\mathrm{mm})\end{array}$ & $\begin{array}{c}\mathbf{T} \\
\text { Media } \\
\text { Anual } \\
\left({ }^{\circ} \mathbf{C}\right) \\
\end{array}$ & Germinación \\
\hline 1 & Lombardía (I) & $45^{\circ} 28^{\prime} \mathrm{N} \quad 9^{\circ} 8^{\prime} \mathrm{E}$ & 1.021 & 13,1 & 43,2 \\
\hline 2 & Toscana (I) & $43^{\circ} 36^{\prime} \mathrm{N} 10^{\circ} 40^{\prime} \mathrm{E}$ & 845 & 14,7 & 58,8 \\
\hline 3 & Eslovenia (SLO) & $46^{\circ} 16^{\prime}$ N 135ㄷ' E & 1.263 & 9,0 & 51,6 \\
\hline 4 & Meseta Castell. (E) & $41^{\circ} 11^{\prime} \mathrm{N} \quad 4^{\circ} 18^{\prime} \mathrm{O}$ & 423 & 11,9 & 72,8 \\
\hline 5 & Andalucía Occ. (E) & $36 \div 39^{\prime} \mathrm{N} \quad 5^{\circ} 41^{\prime} \mathrm{O}$ & 707 & 17,2 & 34,0 \\
\hline 6 & Sierra Morena $(E)$ & $37^{\circ} 51^{\prime} \mathrm{N} \quad 6^{\circ} 17^{\prime} \mathrm{O}$ & 585 & 16,7 & 71,6 \\
\hline
\end{tabular}

Precipitación y temperatura media anual en origen (Hijmans et al., 2005) y tasa de germinación en vivero en Chile (Loewe et al., 1998). 
El ensayo fue establecido en un sitio ubicado en la comuna de Casablanca, provincia y región de Valparaíso (33ํ2' 38" LS, 71ํ 19' 20" LO y 330 m de altitud), a unos $70 \mathrm{~km}$ al oeste de Santiago de Chile. Corresponde a una zona agrícola dedicada en la actualidad mayoritariamente a la viticultura de regadío intensiva. La vegetación de la comuna se caracteriza por su riqueza en bosque nativo esclerófilo, que permanece principalmente en los sectores altos de las planicies litorales y de la cordillera costera, así como en quebradas húmedas y cursos de agua. También se destacan plantaciones de pino y eucalipto, las que sin embargo sufren cada año incendios forestales que en esta zona son frecuentes en la época de verano (e-Casablanca, 2010).

Casablanca posee un clima de tipo mediterráneo, con veranos de sequía prolongada y lluvias invernales cortas e intensas. Las temperaturas medias de la zona, desde el establecimiento del ensayo en 1994 hasta el 2009, presentan una diferencia moderada de temperatura entre estaciones del año, con $15 \stackrel{\circ}{\circ}$ en verano y $10 \stackrel{\circ}{C}$ en invierno. Sin embargo, la oscilación térmica diaria es amplia, con una media de las máximas y de las mínimas para la estación cálida de 29 y de $3,3{ }^{\circ} \mathrm{C}$ y para la estación fría de 26 y de $-1,3{ }^{\circ} \mathrm{C}$, respectivamente. En relación a las precipitaciones, el promedio anual es de $392 \mathrm{~mm}$, de los cuales solo $23 \mathrm{~mm}$ caen en verano. Cabe destacar la irregularidad de las precipitaciones anuales, que oscilaron entre 79 y $887 \mathrm{~mm}$ en los años del estudio. La duración promedia de la época seca es de 4,5 meses, aunque puede variar entre 3 a 8 meses (Cuadro $\mathrm{N}^{\circ} 2$ ). La cantidad de horas con neblina es en promedio superior a 3.500 horas anuales.

\section{Cuadro $\mathrm{N}^{\circ} 2$ \\ SERIES DE TEMPERATURAS Y PRECIPITACIÓN REGISTRADAS EN CASABLANCA DURANTE PERÍODO 1994-2009}

\begin{tabular}{|c|c|c|c|c|c|}
\hline \multirow[b]{2}{*}{ Año } & \multicolumn{3}{|c|}{ Temperatura } & \multirow{2}{*}{$\begin{array}{l}\text { Precipitación } \\
\text { (mm/año) }\end{array}$} & \multirow{2}{*}{$\begin{array}{c}\text { Meses } \\
\text { Secos } \\
\left(N^{\circ}\right) \\
\end{array}$} \\
\hline & $\begin{array}{c}\text { Mín. } \\
\text { Absoluta } \\
\left({ }^{\circ} \mathrm{C}\right)\end{array}$ & $\begin{array}{c}\text { Máx. } \\
\text { Absoluta } \\
\left({ }^{\circ} \mathbf{C}\right)\end{array}$ & $\begin{array}{l}\text { Media } \\
\left({ }^{\circ} \mathrm{C}\right)\end{array}$ & & \\
\hline 1994 & $-0,04$ & 26,50 & 12,50 & 258 & 6 \\
\hline 1995 & 0,45 & 27,73 & 12,08 & 263 & 4 \\
\hline 1996 & 0,30 & 28,09 & 11,87 & 256 & 4 \\
\hline 1997 & 1,55 & 28,27 & 13,23 & 887 & 3 \\
\hline 1998 & 1,53 & 28,84 & 12,59 & 79 & 8 \\
\hline 1999 & 0,88 & 26,65 & 12,28 & 332 & 3 \\
\hline 2001 & 0,77 & 26,63 & 12,15 & 451 & 6 \\
\hline 2002 & 1,49 & 26,83 & 12,13 & 726 & 4 \\
\hline 2003 & 1,27 & 26,54 & 12,43 & 419 & 4 \\
\hline 2004 & 1,66 & 27,02 & 12,54 & 368 & 3 \\
\hline 2005 & 1,18 & 25,93 & 13,15 & 473 & 3 \\
\hline 2006 & 1,51 & 29,01 & 14,48 & 476 & 5 \\
\hline 2007 & $-0,54$ & 26,00 & 12,20 & 182 & 6 \\
\hline 2008 & 1,70 & 26,76 & 13,87 & 466 & 5 \\
\hline 2009 & 0,43 & 28,53 & 13,72 & 241 & 4 \\
\hline Media & 0,94 & 27,3 & 12,7 & 392 & 4,5 \\
\hline
\end{tabular}

(Fuente: Dirección Meteorológica de Chile, 2010)

El sitio del ensayo es llano con una ligera ondulación del terreno, pertenece a la serie de suelos Las Rosas LRR (Franco Arenoso Fino) y no había sido cultivado en los años previos a la

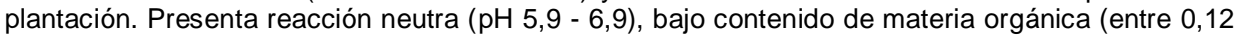
y $1,21 \%$ ) y no es salino (C.E. mmho/cm entre 0,33 y 0,51 ). Su disponibilidad de nitrógeno y fósforo es baja (6-10 ppm y 4-10 ppm, respectivamente), a diferencia de la disponibilidad de potasio, que es media (23-104 ppm). Para la instalación del ensayo, el suelo se preparó con un laboreo completo y subsolado somero sobre la línea de plantación a una profundidad de $30-40 \mathrm{~cm}$. La plantación se realizó en Agosto de 1994, utilizando plantas en macetas de 2 años de edad, con una altura promedio de unos $40 \mathrm{~cm}$ (Loewe et al., 1998). Se proporcionó un riego de establecimiento de 
5 L/planta y se fertilizó de acuerdo a las carencias nutricionales detectadas, con urea ( $35 \mathrm{~g} / \mathrm{planta}$ aplicada en 2 zanjas paralelas separadas a $30 \mathrm{~cm}$ de la planta a una profundidad de $5 \mathrm{~cm}$ ). Los tratamientos culturales repetidos a lo largo de los primeros 15 años consistieron en control de la maleza (años 1, 3 y 4), podas de formación, que consideraron selección de ápices, poda de balance en individuos inclinados y eliminación de ramas más gruesas en algunos verticilos (años 5 , 7,11 y 15), la primera incluyó además la eliminación total de las ramas juveniles con crecimiento averticilado; abonado (años 3, 4 y 5), más podas de levante del fuste (años 8 y 15), en esta última ocasión en combinación con un clareo sistemático que extrajo aproximadamente la mitad de los pies vivos.

El diseño experimental del ensayo fue de tres bloques completos al azar, con 294 plantas cada uno en 6 parcelas de $7 \times 7$ plantas, correspondientes a las 6 procedencias. En total, fueron 882 plantas más una fila de borde, con un espaciamiento de $2 \times 3 \mathrm{~m}$ (1.667 pies/ha), ocupando en total una superficie de menos de 1 hectárea. Desde 1994 hasta 2009, se midó repetidamente el diámetro (DAP) y la altura total de cada árbol. La colocación de los tres bloques pretendía captar el principal gradiente de variación del terreno, asociado a una suave vaguada que recorre el ensayo longitudinalmente.

Sin embargo, desde los primeros años de desarrollo se observó heterogeneidad dentro de los bloques, con zonas o manchas con árboles de peor arraigo y desarrollo, debido aparentemente a cambios de suelo. Por ello, a la hora de evaluar el crecimiento medio de cada procedencia, se realizó un análisis post-hoc mediante la corrección de la variable estudiada por la media de residuos en un vecindario conformado por los árboles más próximos de cada árbol target, o análisis espacial iterativo móvil (Bartlett, 1978; Wilkinson et al., 1983; Loo-Dinkins, 1992; Anekonda y Libby, 1996; Joyce et al., 2002; Mutke et al., 2007, 2012; Zas, 2008).

El vecindario alrededor de cada target usado para calcular el valor de la media de los residuos de los vecinos fue un rectángulo de hasta ocho posiciones vecinas adyacentes, aunque debido a la presencia masiva de marras, en algunas zonas se amplió a un vecindario romboidal de doce árboles.

La media de los residuos del vecindario fue usada como covariable para ajustar variabilidad espacial en parámetros dasométricos a los 15 años de edad. El valor fenotípico de cada variable se modeló a través de cuatro componentes aditivas: la media general, el efecto del Grupo geográfico en origen $i$ (longitud oeste o este), la procedencia $j$ dentro de su Grupo y la covariable espacial estimada iterativamente, más un término de error aleatorio:

$$
\begin{array}{ll}
H_{j(i) x y}= & \mu+G_{i}+P_{j(i)}+\sum^{n} \gamma_{k} M A_{k x y}+\varepsilon_{x y} \\
\text { Donde } & \begin{array}{l}
H_{j(i) x y}: \quad \text { Altura del árbol de procedencia } j \text { del grupo geográfico } i \text { en } \\
\text { la posición }(x, y)
\end{array} \\
\mu: \quad \begin{array}{l}
\text { Media general } \\
G_{i}: \text { Efecto del grupo geográfico en origen } i(\text { Oeste/Este) }
\end{array} \\
P_{j(i)}: \quad \begin{array}{l}
\text { Efecto de la procedencia } j \text { dentro del grupo geográfico } i \\
\gamma_{k}:
\end{array} \quad \begin{array}{l}
\text { Eefecto lineal de la covariable } M A_{k x y}, \text { la media móvil de los k- ésimos } \\
\text { residuos }
\end{array} \\
\varepsilon_{x y):} \text { E error residual en la posición }(x, y)
\end{array}
$$

El mismo modelo se ajustó también para el diámetro normal, medido en el mismo inventario, aunque conviene tener en cuenta que altura y diámetro guardaban una alta correlación $(r=0,9)$. 


\section{RESULTADOS}

Durante los primeros seis años, cuatro de ellos muy secos, con menos de $270 \mathrm{~mm}$ de lluvia anual, el ensayo presentó crecimientos muy limitados (altura media de $62 \mathrm{~cm}$ en 1999 , equivalente a un incremento anual de $12,4 \mathrm{~cm}$ ), retrasando el cambio de fase desde el estado juvenil inicial. No obstante, tras el cambio de fase vegetativa a adulto con crecimiento verticilado y con tipo de acículas definitivo, junto a las intervenciones de manejo realizadas, el ensayo presentó un incremento notable en altura, alcanzando en 2009 una media de $3,80 \mathrm{~m}$, equivalente a un incremento medio anual de $25,3 \mathrm{~cm}$, más del doble del registrado en los primeros años (Figura 1); el diámetro medio a la altura del pecho superó los $10 \mathrm{~cm}$ en las tres procedencias españolas, frente al valor más bajo, $7 \mathrm{~cm}$, correspondiente a Lombardía.

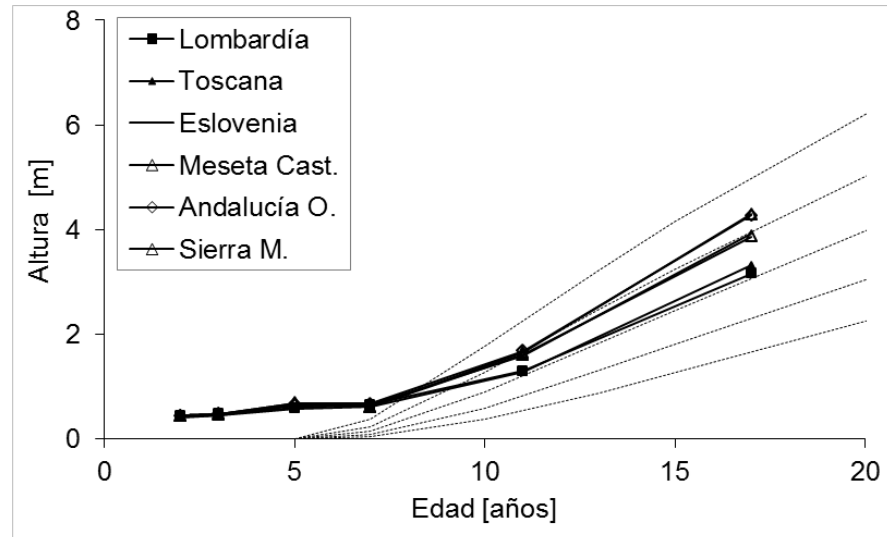

Para comparar, se añade en trazado discontinuo las curvas de calidad españolas de la especie para alturas dominantes de $13,15,17,19$ y $21 \mathrm{~m}$ a los 100 años (Calama et al. 2003), desplazadas 5 años a la derecha para tener en cuenta el retraso inicial del ensayo.

\section{Figura $\mathbf{N}^{\circ} 1$ \\ ALTURAS MEDIAS DE LAS PROCEDENCIAS ENTRE 1994 Y 2009 SEGÚN EDAD DEL ENSAYO}

En cuanto a la supervivencia, las procedencias italianas presentaron más marras (Toscana $48 \%$ y Lombardía 34\% del total) frente a las procedencias Meseta Castellana, Andalucía Occidental y Eslovenia, cada una con un 22\%, y Sierra Morena con un 29\%.

Se observó autocorrelación espacial en el tamaño del arbolado a pequeña escala entre y dentro las unidades experimentales contiguas del ensayo (Figura $\mathrm{N}^{\circ} 2 \mathrm{a}$ ). De hecho, un modelo aditivo para la altura del árbol a los 15 años, que tuviera en cuenta solamente los efectos aditivos de la procedencia $i$ y del bloque $j$, no sería capaz de captar esta variación espacial correctamente, violando en consecuencia los supuestos básicos de independencia espacial de los términos de error (Figura 2.b).

El ajuste iterativo por las medias móviles de los residuos de los vecinos más próximos arrojó un modelo con $\mathrm{R}^{2}$ de 0,88 , atribuyendo el $77 \%$ de la variación de altura entre árboles al efecto de su posición en la parcela (Figura $\mathrm{N}^{\circ} 2 \mathrm{2}$ ) frente a un $8 \%$ debido a las diferencias entre grupos y $3 \%$ de la procedencia dentro de cada grupo, sumando estas dos un $11 \%$ (proporción de 
suma de cuadrados, Cuadro $\mathrm{N}^{\circ} 3$ ). La procedencia eslovena fue la de menor altura, y las españolas las de mayor (Cuadro $\mathrm{N}^{\circ} 4$ ).

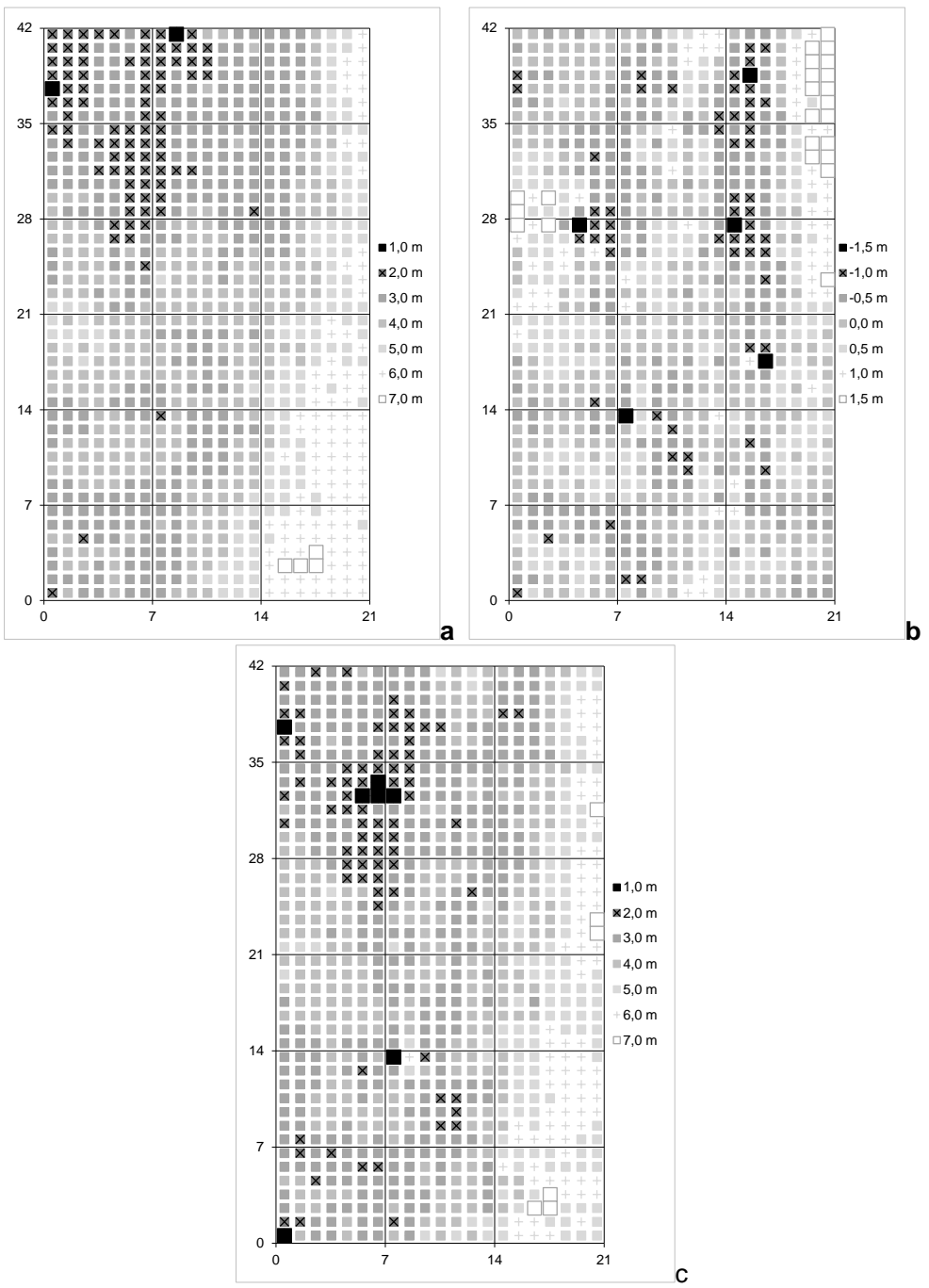

a. Altura del arbolado a los 15 años de edad, que revela un gradiente diagonal a los tres bloques longitudinales del ensayo (filas 1-7, fila 8-14, filas 15-21, respectivamente) que traspasa las unidades experimentales de $7 \times 7$ árboles de la misma procedencia. b. Residuos del modelo aditivo para la altura de cada árbol según procedencia y bloque, persistiendo la autocorrelación espacial.

c. Efecto espacial, estimado a partir de los ajustes iterativos por los residuos de los vecinos más próximos.

Figura $\mathrm{N}^{\circ} 2$

MEDIA MÓVIL LOCAL (3 X 3 POSICIONES) 
Cuadro $\mathrm{N}^{\circ} 3$

ANÁLISIS DE VARIANZA PARA LA ALTURA DEL ÁRBOL A LOS 15 AÑOS, AGRUPANDO LAS 6 PROCEDENCIAS EN DOS GRUPOS, OESTE (ESPAÑA) Y ESTE (ITALIA Y SLOVENIA)

\begin{tabular}{|l|r|r|r|r|}
\hline Fuente de Variación & $\begin{array}{c}\text { Suma de } \\
\text { Cuadrados }\end{array}$ & $\begin{array}{c}\text { Grados de } \\
\text { Libertad }\end{array}$ & $\begin{array}{c}\text { Cuadrado } \\
\text { Medio }\end{array}$ & \multicolumn{1}{c|}{ Valor F } \\
\hline Grupo & 33,0 & 1 & 33,0 & 188 \\
Procedencia/Grupo & 13,5 & 4 & 3,4 & 19 \\
$\mathrm{MA}_{1}$ & 263,3 & 1 & 263,3 & 1.504 \\
$\mathrm{MA}_{2}$ & 63,8 & 1 & 63,8 & 364 \\
$\mathrm{MA}_{3}$ & 9,2 & 31 & 9,2 & 52 \\
Residuos & 54,1 & 309 & 0,2 & \\
\hline Total & $\mathbf{4 3 6 , 8}$ & $\mathbf{3 1 7}$ & \multicolumn{2}{|r}{} \\
\cline { 1 - 3 } & & &
\end{tabular}

Las covariables del ajuste espacial iterativo $\mathrm{MA}_{\mathrm{i}}$ son las medias móviles de los residuos de los vecinos más próximos. El p-valor de todas las fuentes de variación fue inferior a 0,0001.

Cuadro $\mathrm{N}^{\circ} 4$

COMPARACIÓN ENTRE ALTURAS MEDIAS AJUSTADAS A LOS 15 AÑOS PARA LAS 6 PROCEDENCIAS ENSAYADAS

\begin{tabular}{|l|l|cr|}
\hline Grupo Geográfico & Procedencia & $\begin{array}{c}\text { Altura } \\
(\mathbf{\pm S E})(\mathbf{m})\end{array}$ \\
\hline \multirow{3}{*}{ Este } & Toscana (I) & $3,74 \pm 0,058$ & bc \\
& Lombardía (I) & $3,39 \pm 0,061$ & bc \\
& Eslovenia (SLO) & $3,09 \pm 0,055$ & d \\
\hline \multirow{3}{*}{ Oeste } & Sierra Morena (E) & $4,11 \pm 0,061$ & a \\
& Andalucía Occ. (E) & $4,02 \pm 0,060$ & a \\
& Meseta Castell. (E) & $3,91 \pm 0,055$ & ab \\
\hline
\end{tabular}

Los valores con la misma letra no difieren significativamente al $5 \%$ (comparaciones múltiples ajustadas por Bonferroni).

El modelo para el diámetro del árbol arrojó un análisis de varianza similar, lo que concuerda con la alta correlación de altura y diámetro.

El ajuste espacial permitió atribuir el $68 \%$ de la variación de altura entre árboles al efecto de su posición en la parcela, frente a un $8 \%$ debido a las diferencias entre los dos grupos y un $2 \%$ de la procedencia dentro de cada grupo geográfico; la variación total entre procedencias está dada entonces por la suma de ambas, equivalente a un $11 \%$.

El único cambio cuantitativo fue la superioridad de la procedencia eslovena dentro del grupo de procedencias orientales, superando a las dos italianas que presentan árboles más delgados, acercándose a las procedencias españolas (Cuadro $N^{\circ} 5$ ). 


\section{Cuadro $\mathrm{N}^{\circ} 5$ \\ COMPARACIÓN ENTRE LOS DIÁMETROS MEDIOS AJUSTADOS A LOS 15 AÑOS PARA LAS 6 PROCEDENCIAS ENSAY ADAS}

\begin{tabular}{|l|l|cr|}
\hline Grupo Geográfico & Procedencia & $\begin{array}{c}\text { DAP } \\
\text { ( } \pm \text { SE) (cm) }\end{array}$ \\
\hline \multirow{3}{*}{ Este } & Eslovenia (SLO) & $9,23 \pm 0,29$ & b \\
& Toscana (I) & $7,54 \pm 0,31$ & c \\
& Lombardía (I) & $6,93 \pm 0,28$ & c \\
\hline \multirow{3}{*}{ Oeste } & Andalucía Occ. (E) & $10,63 \pm 0,31$ & a \\
& Sierra Morena (E) & $10,19 \pm 0,28$ & ab \\
& Meseta Castell. (E) & $10,08 \pm 0,30$ & ab \\
\hline
\end{tabular}

Los valores con la misma letra no difieren significativamente al $5 \%$

(comparaciones múltiples ajustadas por Bonferroni).

\section{DISCUSIÓN}

En general, el pino piñonero europeo ha mostrado una buena adaptación a las condiciones del sitio del ensayo en la zona central de Chile, aunque una serie inicial de años secos a muy secos ha supuesto un estrés tras plantación considerable, causando un retraso en el cambio de la fase vegetativa y posiblemente en el establecimiento del sistema radicular en el terreno. El desarrollo del ensayo tras esos inicios muy adversos ha sido alentador, con una supervivencia de $52-78 \%$ de los árboles y la recuperación de un buen patrón de crecimiento en altura después de 1999, año en que se realizó una poda de formación que tuvo un efecto visible y significativo en el desarrollo del rodal. Cabe notar que en las mismas condiciones, un ensayo de algarrobo europeo establecido el mismo año en un sector adyacente murió debido a la reducida disponibilidad hídrica.

El mejor desarrollo de las procedencias españolas se podría relacionar con la mayor aridez de su clima en origen, que les habría permitido adaptarse para resistir mejor en condiciones extremas, mientras que el menor crecimiento en altura de la procedencia Eslovenia, la más templada y húmeda de todas, podría tener su relación con un sitio de plantación fuera del rango natural de dicha procedencia. De todas formas, se considera que el reducido número de procedencias incluidas en este ensayo no permite obtener recomendaciones definitivas respecto a las procedencias más recomendables, teniendo además en cuenta que el sitio de ensayo representa probablemente el margen árido de las zonas potenciales para el cultivo de esta especie en Chile (Loewe y González, 2003).

Al comparar los resultados obtenidos en este ensayo con las curvas de crecimiento medidas en una repoblación de Pinus pinea en la Reserva Nacional Lago Peñuelas (Loewe et al,. 1998), en un sector costero de la misma región ubicado a $13 \mathrm{~km}$ del ensayo, de mayor influencia oceánica, donde se dan condiciones para masas predominantes de eucalipto y pino radiata, se observa que en esta última localidad los crecimientos fueron muy superiores, alcanzando $16 \mathrm{~cm}$ de diámetro y $9,9 \mathrm{~m}$ de altura media a los 17 años, y $30 \mathrm{~cm}$ y $23 \mathrm{~m}$ a los 40 años. El crecimiento en altura reportado para la especie en esta y también en otras localidades más australes de Chile es muy superior a las calidades de estación españolas (Loewe et al,. 1998). En Argentina, Calderón et al. (2004) clasifican a Pinus pinea como una especie apta para la zona de Mendoza, bajo riego, donde se obtuvo a los 14 años alturas medias de 5,9-6,7 m y diámetros medios de $16-28 \mathrm{~cm}$. No obstante Mendoza presenta suelos de mayor fertilidad, y en este caso riego, los incrementos no son tan disimiles como se hubiera esperado de situaciones con tan diferente régimen hídrico. En este sentido, los resultados obtenidos en el ensayo aquí analizado, donde la especie no ha crecido tan bien como en otros sitios del Cono Sur de América, confirman el elevado potencial de adaptación de la especie en diferentes ambientes de esta región. 
La importante variación espacial observada dentro del ensayo (Cuadros $\mathrm{N}^{\circ} 3$ y $\mathrm{N}^{\circ} 4$ ), tanto en diámetro como en altura, confirma que los crecimientos obtenidos responden a la condición del micrositio. Este fenómeno de fuertes autocorrelaciones espaciales es muy común en ensayos con esta especie, reflejo de su sensibilidad a factores edáficos (Court-Picon et al., 2004; Mutke et al, 2007, 2010 y 2012) y a la elevada variación de los factores edáficos en superficies reducidas de gran parte de los suelos de Chile. Las magnitudes de la variación entre alturas individuales atribuidas a este efecto y a la procedencia, y la predominancia absoluta de la primera son similares a resultados de otros ensayos de procedencias (Gordo et al,. 2007; Mutke et al., 2010). Estas conclusiones concuerdan con los trabajos moleculares sobre isoenzimas y ADN de cloroplasto o nuclear, que encontraron una muy baja diferenciación genética entre procedencias de esta especie, e incluso entre países de origen alejados (Fallour et al., 1997; Fady et al., 2008; Vendramin et al., 2008; Mutke et al., 2011).

Respecto de las labores culturales aplicadas, se observa una preponderancia del efecto positivo de la poda de formación inicial por sobre la fertilización, control de maleza y poda de levante. Esta práctica modificó la tendencia de los crecimientos principalmente en diámetro, aunque también en altura, lo que ha sido observado en otras situaciones en Chile. La eliminación de las ramas juveniles inferiores es recomendable en situaciones en que el pino piñonero presenta crecimientos iniciales reducidos.

En general se considera que el cultivo de la especie sería plenamente viable en sitios adyacentes y similares de la región de Valparaíso, así como en otros del secano costero e interior con características similares a la localización del ensayo, o más favorables, donde podría integrarse como elemento arbóreo de interés en sistemas agroforestales.

Se aprecia un buen potencial para plantaciones extensivas de esta especie en áreas marginales para cultivos agrícolas (pendientes excesivas, falta de riego) y para eucalipto o pino radiata (por sequía, riesgo de heladas), especies forestales tradicionalmente empleadas en Chile en cultivos tanto intensivos como extensivos.

Se considera que el pino piñonero en áreas similares a las del ensayo en la región podrá cumplir un rol fundamentalmente de protección ambiental, y de producción frutal contenida, aunque bajo riego la producción de piñones podría alcanzar niveles de productividad interesantes.

\section{RECONOCIMIENTOS}

El presente estudio se enmarca en el proyecto "El piñón comestible del pino piñonero (Pinus pinea L.), un negocio atractivo para Chile" (2008-2011), financiado por INNOVA-CORFO y por el sector privado. Se agradece a la Universidad Iberoamericana de Ciencias y Tecnología por ceder el terreno para el ensayo.

\section{REFERENCIAS}

Albert , F., 1909. Los 7 árboles forestales más recomendables para el país. Imprenta Cervantes. 52.

Anekonda, T. \& Libby, W., 1996. Effectiveness of nearest-neighbor data adjustment in a clonal test of redwood. Silvae Genet 45(1): 46-51.

Anónimo, 1957. El Plan Chillán, realización y promesa. Boletín № 9.

Badal, E., 2001. La recolección de piñas durante la prehistoria en la Cueva de Nerja (Málaga). 101 104. In: V. Villaverde (ed.), De neandertales a cromañones -el inicio del poblamiento humano en las tierras valencianas. Universitat de València, Spain. 
Bartlett, M., 1978. Nearest Neighbour models in the analysis of field experiments. J R Statist Soc B 40(2): 147174.

Calama, R., Cañadas, N. \& Montero, G., 2003. Inter-regional variability in site index models for Even aged stands of Stone Pine (Pinus pinea L.) in Spain, Ann. For. Sci. 60: 259-269.

Calderon, A., Pérez, S., Riu, N., Settepani, V. \& Bustamante, J., 2004. Comportamiento de coníferas bajo riego Mendoza (Argentina). Rev. FCA UN Cuyo Tomo XXXVI, año 2: 1-6.

Court-Picon, M., Gadbin-Henry, C., Guiba, L. F, \& Roux, M., 2004. Dendrometry and morphometry of Pinus pinea L. in Lower Provence (France): adaptability and variability of provenances. For. Ecol. Manag. 194: $319-333$.

E-CASABLANCA, 2010. Características comunales. (En http://www.ecasablanca.cl/webcb/index.php?option=com_phocadownload\&view=category\&id=5:casablanca\&ltmid $=59$ (accedido Febrero 28, 2009).

Fady, B., Fineschl, S. \& Vendramin, G., 2008. Pino piñonero. Pinus pinea. EUFORGEN. Guía técnica para la conservación genética y utilización del pino piñonero (Pinus pinea).

Fallour, D., Fady, B. \& Lefevre, F., 1997. Study of Isozyme Variation in Pinus pinea L.: Evidence for Low Polymorphism. Silvae Geneticae 46: 201-207.

Gil, L., 1999. La transformación histórica del paisaje: La permanencia y la extinción local del pino piñonero. En: Marín F., Domingo J., Calzado A. (eds.): $1^{\text {as }}$ jornadas de historia, socio economía y política forestal (1997). Los montes y su historia - una perspectiva política, económica y social. Universidad de Huelva, 151-185.

Gordo, J., Mutke, S. \& Gil, L., 2007. Ausencia de diferenciación ecotípica entre rodales selectos de pino piñonero en la cuenca del Duero. Inv. Agrar. Sist. Rec. Forest. 16(3): 253-261.

Hijmans, R., Cameron, S., Parra, J., Jones, P. \& Jarvis, A., 2005. Very high resolution interpolated climate surfaces for global land areas. International Journal of Climatology 25: 1965-1978.

Joyce, D., Ford, R. \& Fu, Y., 2002. Spatial patterns of tree height variations in a Black Spruce farm-field progeny test and neighbours-adjusted estimations of genetic parameters. Silvae Genet 51(1), 13-18.

Loewe, V., Toral, M., Delard, C., López, C. \& Urquieta, E., 1998. Monografía de Pino piñonero (Pinus pinea). CONAF-INFOR-FIA, 100.

Loewe, V. \& González, M., 2003. Sicomoro, grevillea, roble rojo americano, pino piñonero, castaño, ruil y cerezo americano, nuevas alternativas para producir madera de alto valor. INFOR-FIA, 320.

Loewe, V. \& González, M., 2007. Pino piñonero: el potencial de su madera y fruto. Chile Forestal 334:49-53.

Loo-Dinkins, J., 1992. Field Test Design. En: Fins L, Friedman ST, Brotschol JV (eds.), Handbook of quantitative forest genetics, Forestry Sciences 39, Kluwer Academics Publishers, Dordrecht: 96139.

Mutke, S., Iglesias, S. \& Gil, L., 2007. Selección de clones de pino piñonero sobresalientes en la producción de piña. Invest. Agr. Sist. Recur. For. 16(1): 39-51.

Mutke, S., Calama, R., González-Martínez, S., Montero, G., Gordo, J., Bono, D. \& Gil, L., 2012. Mediterranean Stone Pine: Botany and Horticulture. En: Janick J. (ed.): Horticultural Review 39. John Wiley \& Sons, Inc., Hoboken, New Jersey: 153-201.

Mutke, S., Fady, B., Ben, A., Khaldi, A., Khouja, M., Gonzales-Martinez, S., Climent, J., Sebastiani, F., Torre, S. \& Vendramin, G., 2011. The further we search, the less we found: low genetic variation in quantitative and molecular traits in Mediterranean stone pine (Pinus pinea). MEDPINE $4^{\text {th }}$ International Conference on Mediterranean Pines "Conservation, Ecology, Restoration and Management of Mediterranean Pines and their Ecosystems: Challenges under global change",

Avignon, 6-10 Junio 2011.

Mutke, S., Gordo, J., Chambel, M., Prada, M., Álvarez, D., Iglesias, S. \& Gil, L., 2010. Phenotypic plasticity is stronger than adaptative differentiation among Mediterranean stone pine provenances. Forest Systems 19(3): 354-366. 
Prada, M., Gordo, J., De Miguel, J., Mutke, S., Catalan, G., Iglesias, S. \& Gil, L., 1997. Las regiones de procedencia de Pinus pinea L. en España. Organismo Autónomo de Parques Naturales, Madrid.

Soto, D., Gysling, J. \& Loewe, V., 2008. Antecedentes del Mercado Internacional de Piñones de Pino. Revista Ciencia e Investigación Forestal. Vol. 14(3): 599-623.

Thirgood, J., 1981. Man and the Mediterranean forest. A history of resource depletion. Academic Press, London.

Vendramin, G., Fady, B., González-Martínez, S., Hu, F., Scotti, I., Sebastiani, F., Soto, A. \& Petit. R., 2008. Genetically depauperate but widespread: the case of an emblematic Mediterranean Pine. Evolution 62(3): 680688.

Wilkinson, G., Eckert, S., Hancock, T., Mayo, O., 1983. Nearest Neighbour (NN) Analysis of Field Experiments. JR Statist Soc B 45(2), 151-211.

Zas, R., 2008. The impact of spatial heterogeneity on selection: a case study on Pinus pinaster breeding seedling orchards. Can. J. For. Res. 38: 114-124. Doi: 10.1139/X07-099. 
